\title{
ANNIHILATORS OF RELATION MODULES
}

Dedicated to the memory of Hanna Neumann

J. N. MITAL and I. B. S. PASSI

(Received 31 May 1972)

Communicated by M. F. Newman

\section{Introduction}

Let $1 \rightarrow R \rightarrow F \stackrel{\alpha}{\rightarrow} G \rightarrow 1$ be a non-cyclic free presentation of a group $G$, $R=R_{1}>R_{2}>R_{3}>\cdots>R_{n}>\cdots$ the lower central series of $R$. Then $R_{n} / R_{n+1}$, $n \geqq 1$, can be regarded as a right $G$-module by defining the action of $G$ via conjugation in $\boldsymbol{F}$. We wish to investigate the annihilators of these modules which we call higher relation modules.

Our main result is that if Ann $R / R_{2}=(0)$, then, for all $n \geqq 1$,

$$
\text { Ann } R_{n} / R_{n+1}=(0) \text { (Ann = annihilator). }
$$

We prove that there always exists an integer $c \geqq 1$, independent of $n$, such that

$$
A_{G}^{c} \cdot \operatorname{Ann} R_{n} / R_{n+1} \leqq \operatorname{Ann} R / R_{2},
$$

where $A_{G}$ is the augmentation ideal of $Z G$, the integral group ring of $G$. If $G$ is periodic, then we prove that

$$
\text { Ann } R_{n} / R_{n+1} \leqq \text { Ann } R / R_{2} \text { for all } n \geqq 1 \text {. }
$$

\section{Free di:-erential calculus}

If $F$ is a free group and $D_{1}, D_{2}, \cdots, D_{n}: Z F \rightarrow Z F$ are right derivations, we

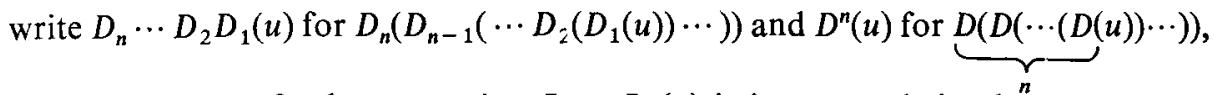
$u \in Z F$. For $n=0$, the expression $D_{n} \cdots D_{1}(u)$ is interpreted simply as $u$.

Let $1 \rightarrow R \rightarrow F \stackrel{\alpha}{\rightarrow} G \rightarrow 1$ be a free presentation of a group $G$. We extend $\alpha$ by linearity to a ring homomorphism $\alpha: Z F \rightarrow Z G$. Let $\varepsilon: Z F \rightarrow Z$ be the unit augmentation.

Lemma 2.1. Let $n, i$ be integers with $n>i \geqq 0, u \in A_{F}^{i}, v \in A_{F}^{n-i-1} A_{R}$, $D_{1}, \cdots, D_{n}: Z F \rightarrow Z F$ arbitrary derivations. Then 
PRoof. Extend $\alpha$ by linearity to $\alpha: Z F \rightarrow Z G$. Let $r \in R_{n}, z \in$ Ann $R_{n} / R_{n+1}$, $\alpha(u)=z, u \in Z F$. Applying $\theta_{n}$ to $r R_{n+1} \cdot z=0$ gives $(r-1) u \in A_{F} A_{R}^{n}$. Since $R \leqq F_{c}, A_{R} \leqq A_{F}^{c}$. Hence $(r-1) u \in A_{F}^{c(n-1)+1} A_{R}$. Therefore, for arbitrary derivations $D_{1}, D_{2}, \cdots, D_{c(n-1)+1}$,

This gives

$$
\alpha D_{c(n-1)+1} \cdots D_{1}((r-1) u)=0[1] .
$$

$$
\begin{gathered}
\alpha\left(D_{c(n-1)+1} \cdots D_{1}(r-1)\right) \alpha(u)+\varepsilon D_{c(n-1)} \cdots D_{1}(r-1) \alpha D_{c(n-1)+1}(u)+\cdots \\
+\varepsilon(r-1) \alpha D_{c(n-1)+1} \cdots D_{1}(u)=0 .
\end{gathered}
$$

Since $r-1 \in A_{R}^{n} \leqq A_{F}^{c n}$,

$\varepsilon D_{k} D_{k-1} \cdots D_{1}(r-1)=0$ for $k<c n$. Hence we get

$$
\alpha D_{c(n-1)+1} \cdots D_{1}(r) \cdot z=0 .
$$

THEOREM 3.2. Let $1 \rightarrow R \rightarrow F \stackrel{a}{\rightarrow} G \rightarrow 1$ be a non-cyclic free presentation of a group $G$. If for every given partial derivation $d: Z F \rightarrow Z F$ we can find an element $r$ of $R$ and a partial derivation $d_{1}: Z F \rightarrow Z F$ such that $\alpha d(r)=0$ and $\varepsilon d_{1}(r) \neq 0$, then

$$
\text { Ann } R_{n} / R_{n+1} \leqq \text { Ann } R / R_{2} \text {. }
$$

Proof. It is enough [4] to prove that for every $z \in \operatorname{Ann} R_{n} / R_{n+1}, s \in R$ and partial derivation $d: Z F \rightarrow Z F, \alpha d(s) \cdot z=0$. Let $s \in R$ and $d$ be a partial derivation. By hypothesis we can find an element $r \in R$ and a partial derivation $d_{1}$ such that $\varepsilon d_{1}(r) \neq 0, \alpha d(r)=0$. If $r=s$, then $\alpha d(s) \cdot z=0$ trivially. Suppose that $r \neq s$. Let $i \geqq 0$ be the least integer such that

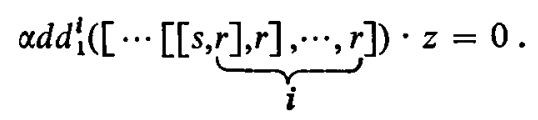

By Lemma 3.1 (case $c=1$ ), this is certainly true for $i=n-1$. This settles the question of the existence of $i$. If $i \neq 0$, then applying Lemma $2.2,(3.3)$ gives

$$
\left\{\varepsilon d_{1}^{i}([\cdots[[s, r], r], \cdots, r]) \alpha d(r)-\varepsilon d_{1}(r) \alpha d d_{1}^{i-1}([\cdots[s, r], \cdots, r])\right\} \cdot z=0 .
$$

But $\alpha d(r)=0, \varepsilon d_{1}(r) \neq 0$. Hence this implies that

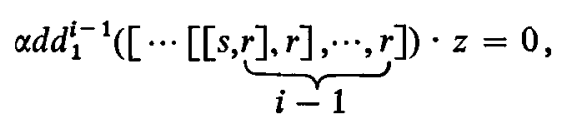

contradicting the minimality of $i$. Hence the least integer $i$ for which (3.3) holds is 0 i.e. $\alpha d(s) \cdot z=0$.

COROLlary 3.4. If $G$ is periodic, then Ann $R_{n} / R_{n+1} \leqq \operatorname{Ann} R / R_{2}$. 
PROOF. Given $d$ to be the partial derivation with respect to the free generator $x_{j}$, say, of $F$, we take $r=x_{i}^{m}$, where $x_{i}$ is a free generator $\neq x_{j}$ and $\alpha\left(x_{i}\right)$ is of order $m$ in $G$. For $d_{1}$ we take the partial derivation with respect to $x_{i}$. This choice of $r$ and $d_{1}$ is possible since $F$ is non-cyclic and $G$ is periodic. Then $\alpha d(r)=0$ and $\varepsilon d_{1}(r)=m \neq 0$. Hence by Theorem 3.2, Ann $R_{n} / R_{n+1} \leqq$ Ann $R / R_{2}$.

Corollary 3.5. If $G$ is finite, then Ann $R_{n} / R_{n+1}=(0)$ for all $n \geqq 1$.

Proof. This follows form Corollary 3.4 and the result that Ann $R / R_{2}=(0)$ when $G$ is finite [5].

LeMma 3.6. Let $R \leqq F_{c}, R \geqq F_{c+1}$. Then for a given $n \geqq 2$

either

or

(i) $R_{i-1} F_{c(i-1)+1}$ and

(ii) $\alpha d_{c(i-1)+1} \cdots d_{1}(s) \cdot z=0$ for every $s \in R_{i}, z \in \operatorname{Ann} R_{n} / R_{n+1}$ and all partial derivations $d_{1}, d_{2}, \cdots, d_{c(i-1)+1}$.

Proof. Let $K$ be the set of natural numbers $i$ which satisfy (b(ii)), By Lemma $3.1, K$ is not empty. Let $i$ be the least member of $K$. If $i=1$, then $\alpha d(s) \cdot z=0$ for every $s \in R, z \in$ Ann $R_{n} / R_{n+1}$ and every partial derivation $d$. Therefore $z \in \operatorname{Ann} R / R_{2}$ [4].

Suppose $i \geqq 2$. We assert that $i$ satisfies (b(i)). For, let $R_{i-1} \leqq F_{c(i-1)+1}$. Since $R \leqq F_{c+1}$ and $R \leqq F_{c}$, we can find an element $r \in R, r \notin F_{c+1}, r \in F_{c}$. By $([1], 4.6)$ it is possible to choose partial derivations $d_{1}, d_{2}, \cdots, d_{c}$ such that

$$
\varepsilon d_{c} d_{c-1} \cdots d_{1}(r) \neq 0 \text {. }
$$

Let $t$ be an arbitrary element of $R_{i-1}$ and $d_{c+1}, d_{c+2}, \cdots, d_{c(i-1)+1}$ be arbitrary partial derivations. Then $[r, t] \in R_{i}$ and, since $i$ satisfies the requirement (b(ii)) of the Lemma, we have

$$
\alpha d_{c(i-1)+1} \cdots d_{1}([r, t]) \cdot z=0
$$

for all $z \in$ Ann $R_{n} / R_{n+1}$. By Lemma 2.2 this gives

$$
\left\{\varepsilon d_{c} \cdots d_{1}(r) \alpha d_{c(i-1)+1} \cdots d_{c+1}(t)-\varepsilon d_{c(i-1)} \cdots d_{1}(t) \alpha d_{c(i-1)+1}(r)\right\} \cdot z=0 .
$$

But $t \in R_{i-1} \leqq F_{c(i-1)+1}$. Hence by $([1], 4.6) \varepsilon d_{c(i-1)} \cdots d_{1}(t)=0$. Also $\varepsilon d_{c} \cdots d_{1}(r) \neq 0$. Hence $\alpha d_{c(i-1)+1} \cdots d_{c+1}(t) \cdot z=0$ for every $t \in R_{i-1}, z \in$ Ann $R_{n} / R_{n+1}$ and arbitrary partial derivations $d_{c+1}, \cdots, d_{c(i-1)+1}$ i.e. $i-1$ satisfies the condition (b(ii)). This contradicts the choice of $i$. Hence it must satisfy (b(i)).

LEMmA 3.7. Let $R \leqq F_{c}, R \leqq F_{c+1}$. Then $\alpha d(r) \cdot z=0$ for all $z \in \operatorname{Ann} R_{n} / R_{n+1}$, $r \in R \cap F_{c+1}$ and all partial derivations $d$. 
Proof. By Lemma 3.6 either 3.6(a) or 3.6(b) holds. If 3.6(a) holds, then there is nothing to prove [4]. Suppose 3.6(b) holds. Choose $s$ in $R_{i-1}$ which is not in $F_{c(i-1)+1}$. By $([1], 4.6)$ we can find partial derivations $d_{1}, \cdots, d_{c(i-1)}$ such that $\varepsilon d_{c(i-1)} \cdots d_{1}(s) \neq 0$. Let $r \in R \cap F_{c+1}$. Then $[r, s] \in R_{i}$ and therefore

$$
\alpha d d_{c(i-1)} \cdots d_{1}([r, s]) \cdot z=0
$$

for all $z \varepsilon \operatorname{Ann} R_{n} / R_{n+1}$ and arbitrary partial derivations $d$. Hence by Lemma 2.2

$$
\left\{\varepsilon d_{c} \cdots d_{1}(r) \alpha d d_{c(i-1)} \cdots d_{c+1}(s)-\varepsilon d_{c(i-1)} \cdots d_{1}(s) \alpha d(r)\right\} \cdot z=0 .
$$

But $r \in F_{c+1}$ and therefore $\varepsilon d_{c} \cdots d_{1}(r)=0$. Also $\varepsilon d_{c(i-1)} \cdots d_{1}(s) \neq 0$. Hence $\alpha d(r) \cdot z=0$ for $r \in R \cap F_{c+1}$ and arbitrary partial derivations $d$.

We can now prove

THEOREM 3.8. There exists an integer $c$, independent of $n$, such that

$$
A_{\mathrm{G}}^{c} \cdot \operatorname{Ann} R_{n} / R_{n+1} \leqq \mathrm{Ann} R / R_{2} \text {. }
$$

Proof. Let $z \in$ Ann $R_{n} / R_{n+1}$. Since $F$ is residually nilpotent, we can find an integer $c \geqq 1$ such that $R \leqq F_{c}, R \leq F_{c+1}$. By Lemma $3.7 \alpha d(s) \cdot z=0$ for all $s \in R \cap F_{c+1}$ and all partial derivations $d$. Let $r \in R$ and $f_{1}, f_{2}, \cdots, f_{c} \in F$. Then $\left[\cdots\left[\left[r, f_{1}\right], f_{2}\right], \cdots, f_{c}\right] \in R \cap F_{c+1}$. Hence

$$
\alpha d\left(\left[\cdots\left[\left[r, f_{1}\right], f_{2}\right], \cdots, f_{c}\right]\right) \cdot z=0 .
$$

It is easy to check that

$$
\alpha d\left(\left[\cdots\left[\left[r, f_{1}\right], f_{2}\right], \cdots, f_{c}\right]\right)=\alpha d(r)\left(\alpha\left(f_{1}\right)-1\right) \cdots\left(\alpha\left(f_{c}\right)-1\right) .
$$

Hence (3.9) gives

$$
\alpha d(r)\left(g_{1}-1\right)\left(g_{2}-1\right) \cdots\left(g_{c}-1\right) \cdot z=0
$$

where $\alpha\left(f_{i}\right)=g_{i}, i=1,2, \cdots, c$. Since this is true for all $r \in R$ and all partial derivations $d$, it follows that $\left(g_{1}-1\right)\left(g_{2}-1\right) \cdots\left(g_{c}-1\right) \cdot z \in$ Ann $R / R_{2}$ [4]. Hence $A_{G}^{c} \cdot \operatorname{Ann} R_{n} / R_{n+1} \leqq \operatorname{Ann} R / R_{2}$.

CoROllary 3.10. If Ann $R / R_{2}=(0)$, then Ann $R_{n} / R_{n+1}=(0)$ for all $n \geqq 1$.

Proof. By Corollary 3.5 we can assume that $G$ is infinite. Since the integral group ring of an infinite group does not have any non-zero element which annihilates its augmentation ideal, the corollary follows from Theorem 3.8.

REMARK 3.11. For a non-cyclic free presentation $1 \rightarrow R \rightarrow F \stackrel{a}{\rightarrow} G \rightarrow 1$ of a group $G$, the annihilator of the relation module $R / R_{2}$ is known $([4],[5])$ to be trivial in the following cases: 
(i) $Z G$ is without zero-divisors;

(ii) the free presentation under consideration is the standard free presentation;

(iii) $G$ is finite;

(iv) $G$ is residually finite and centre of $G$ is infinite;

(v) $G$ is nilpotent;

(vi) the rank of $F$ is infinite.

\section{References}

[1] R. H. Fox, 'Free differential calculus I Derivations in the free group ring', Ann. of Math. (2) 57 (1953), 547-560.

[2] K. W. Gruenberg, Cohomological topics in group theory, Lecture Notes in Mathematics, No. 143, (Springer-Verlag, 1970.)

[3] W. Magnus, A. Karrass, and D. Solitar, Combinatorial group theory, (Interscience Publishers, 1966.)

[4] J. N. Mital, 'On residual nilpotence', J. London Math. Soc. (2) 2 (1970), 337-345.

[5] I. B. S. Passi, and R. C. Singal, Relation modules (unpublished).

Kurukshetra University

Kurukshetra (Haryana), India

and

University of Alberta

Edmonton (Alberta), Canada. 